\title{
Mitigate the dominating signals for super dense optical communication using $\mathrm{HOA}$
}

\author{
Chakresh Kumar ${ }^{1}$ (D) . Ghanendra Kumar ${ }^{2}$
}

(c) Springer Nature Switzerland AG 2019

\begin{abstract}
In this paper, impact of crosstalk and fiber nonlinearities has investigated to enhance the signals strength for 260 channels with the data rate of $10 \mathrm{Gbps}$. Further, power amplification has also maintained with the arrangement of RAMAN-EDFARAMAN hybrid optical amplifier for transmission distance of $65 \mathrm{~km}$. Furthermore, analysis has also observed in terms of received crosstalk and different power level of fiber nonlinearities for channel range from 1 to 260 . Channel spacing is also main concerned for super dense optical communication which has also resolved in this proposed simulation model. In this way, it is recommended that this proposed model is really helped out to the network operator to enhance the future super dense optical commutation process.
\end{abstract}

Keywords RAMAN ·EDFA · HOA · Crosstalk · Fiber nonlinearities · Super dense system

\section{Introduction}

Optical communication data rate up to $100 \mathrm{Gbps}$ has been demonstrated to control the universal telecommunication traffic. The major challenges with the high spectral data transmission are to get the desired parameters with tolerating effect of fiber nonlinearities and crosstalk [1-4]. Least effect of fiber nonlinearities, dispersion and crosstalk are also supported to optical signal to noise ratio (OSNR) which is main acceptable aspect for super dense optical communication system [5-9]. Today's super dense optical communication losses of $0.15 \mathrm{~dB} / \mathrm{km}$ has been attained with typical effective area of $105 \mu \mathrm{m}^{2}$ which has also shown the limited effects of macro and micro bending losses with the support of single mode fiber (SMF) [10-12]. In super dense long distance optical communication, Kerr fiber nonlinearity is the main dominating effect which was resolved with the implementation of coherent compensation techniques [13]. Digital back transmission (DBT) is the most acceptable technique to control the fiber nonlinearities, which is placed near the transmitter and receiver side before the distortion [14]. On the other hand, lower order perturbation compensation is also the superior approach to mitigate the effect of intra and inter channel losses for upgrade the signals strength for super dense optical communication [15]. In the couple of years, some of the research group has developed best acceptable techniques to control the dominating effect of dispersion and power reduction for dense communication, which has really supported to this research work [16-18]. So far, the details knowledge of the above mentioned techniques are required to enhance the signals strength and all the above proposed techniques has also been implemented for wavelength division multiplexing system, no use in super dense optical communication is shown till now for the future super dense optical aspects which is also main concerned. So this paper has considered all the major aspects for proposed super dense

Chakresh Kumar, chakreshk@gmail.com | 1 University School of Information Communication and Technology, Guru Gobind Singh Indraprastha University, New Delhi 110078, India. ${ }^{2}$ Department of Electronics and Communication Engineering, National Institute of Technology, New Delhi, Delhi 110040, India. 
optical communication and presentation of this research work is also given in four sections. First, second, and third section are given the details knowledge of introduction, proposed simulation setup, and analysis and discussions. Further, final conclusion is also explained in section four respectively.

\section{Proposed simulation setup}

Simulation setup for analysis the effect of crosstalk and fiber nonlinearities for $260 \times 10 \mathrm{Gbps}$ super dense communication system is shown in Fig. 1. Channels are arranged with channel spacing of $100 \mathrm{GHz}$ to neglect the effect of intersymbol interference (ISI) which also leads to dispersion and crosstalk. Optical signals are received with the support of data source, $R Z$ electrical drive, sin-square modulator, and CW laser source for one transmitter. This process is also repeated for rest of the transmitters to get the optical signals. Converted optical signals are combined with super dense combiner, which is also shown in Fig. 1. Power meter, optical spectrum analyzer and other measuring instruments are also used during the communication process to observe the signals characteristics.

Two sets of RAMAN-EDFA-RAMAN hybrid optical amplifier are also placed in optical medium with fiber length of $65 \mathrm{~km}$ to maintain the uniform power level throughout the super dense communication process to neglect the effect of fiber nonlinearities and crosstalk.

Use of attenuator and fiber Bragg grading (FBG) are the main instruments to mitigate the effect of crosstalk and fiber nonlinearity which are arranged near the receiver section. Received optical super dense signals are separated out with the support of super dense optical separator which further link to PIN photo diode and low pass filter to get the original electrical signals to observe the effect of crosstalk and fiber nonlinearities with the support of different measuring instruments.

\section{Analysis and discussions}

Final simulation results are shown from Figs. 2, 3, 4 and 5 to get the acceptable rating outcomes for the betterment of super dense communication system. Figure 2 is shown the outcomes in terms of crosstalk with respect to number of channels with the condition of different channels spacing. Further, recorded value are given as -60 to $-1 \mathrm{~dB}$ for $100 \mathrm{GHz}$ channel spacing, -80 to $-8 \mathrm{~dB}$ for $50 \mathrm{GHz}$ channel spacing, -87 to $-12 \mathrm{~dB}$ for $25 \mathrm{GHz}$ channel spacing, -90 to $-15 \mathrm{~dB}$ for $12.5 \mathrm{GHz}$ channels spacing, and -90 to $6.25 \mathrm{~dB}$ for $6.25 \mathrm{GHz}$ channels spacing respectively.

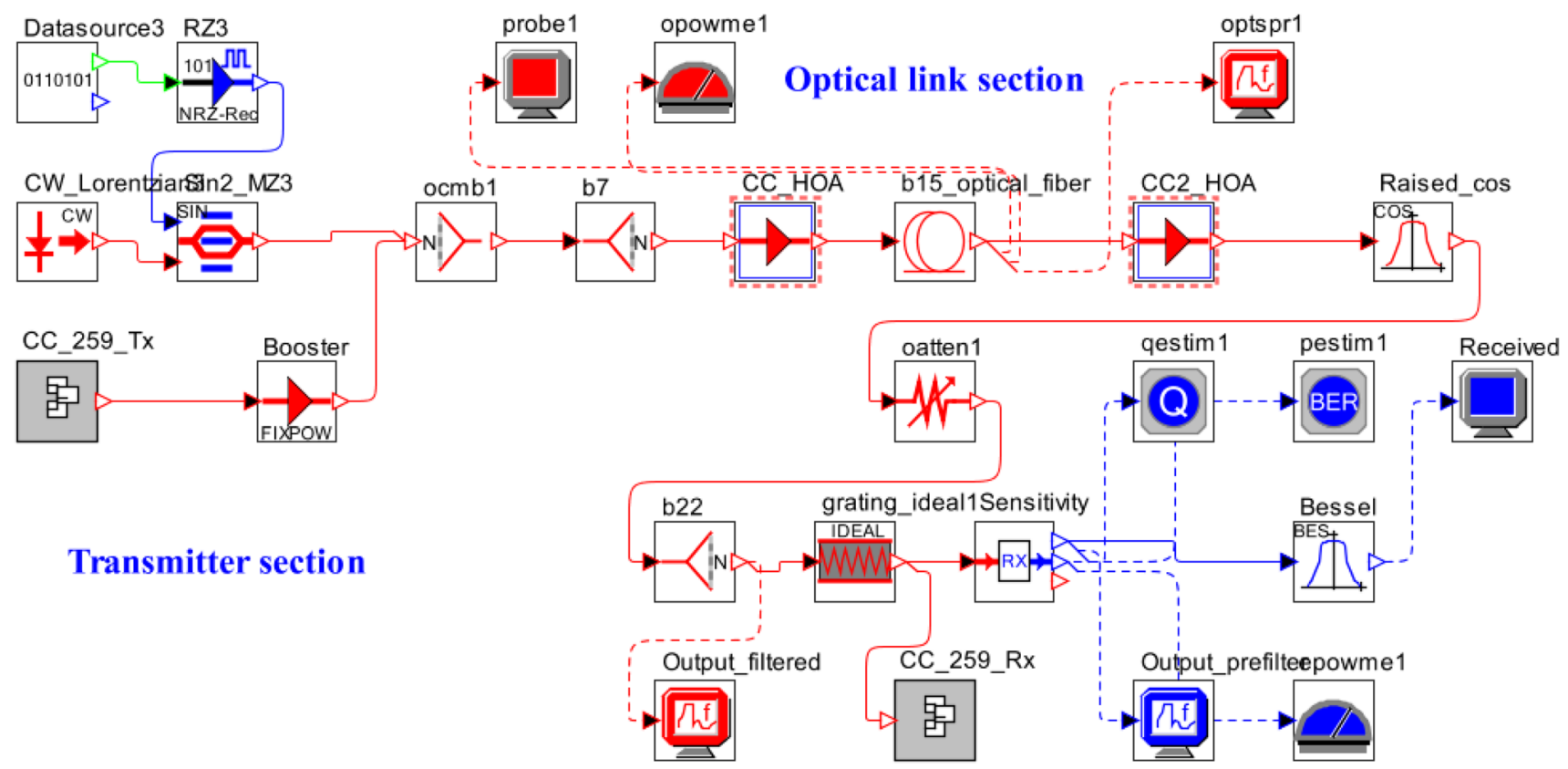

Receiver section

Fig. 1 Simulation setup to analysis the impact of crosstalk and fiber nonlinearities 


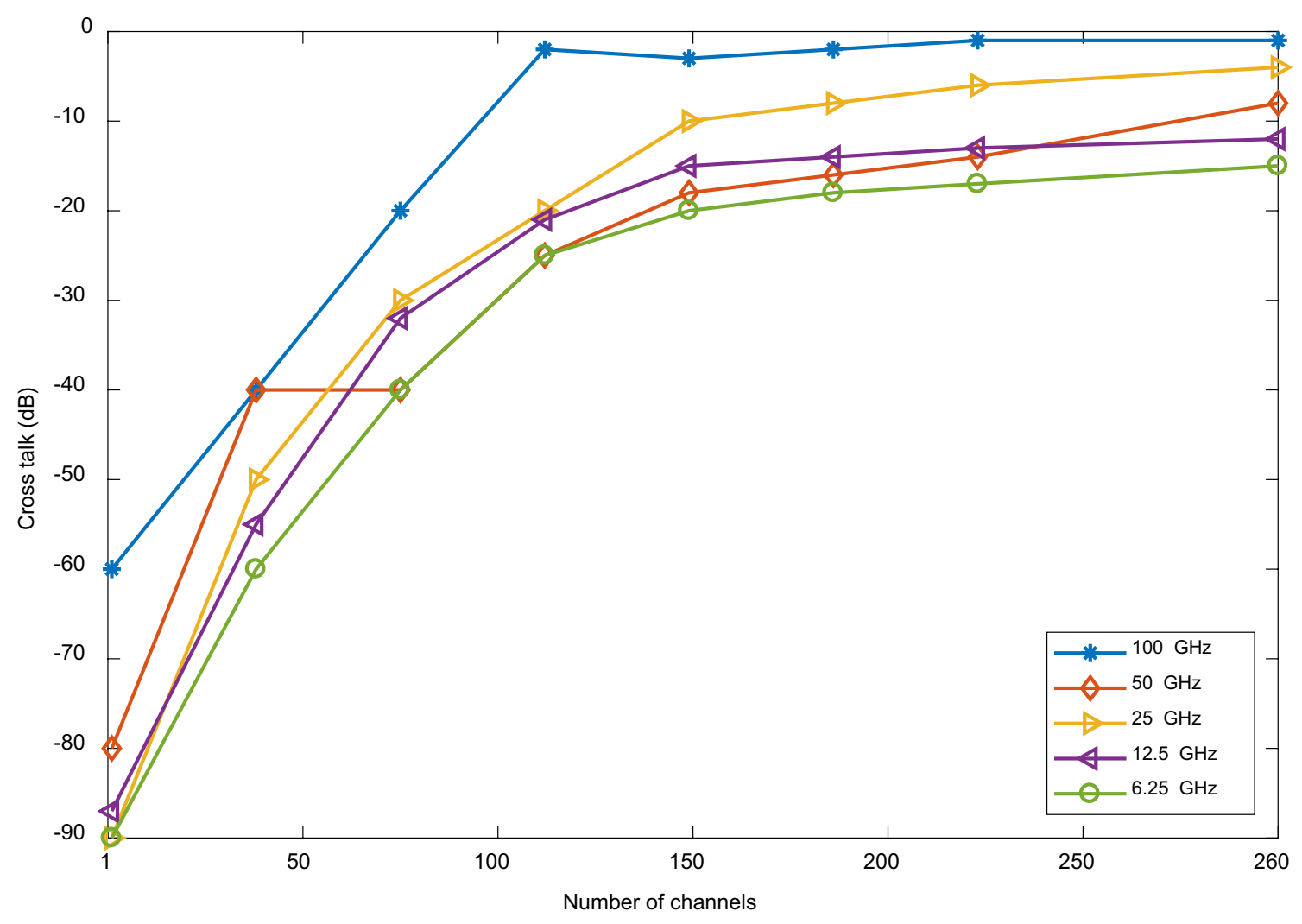

Fig. 2 Crosstalk with respect to number of channels

In the similar manner, power level of different fiber nonlinearities is observed from Fig. 3. The noticed value are given as 12.5 to $32.5 \mathrm{~dB}$ for SBS, $13.835 .8 \mathrm{~dB}$ for SRS, -39 to $-1 \mathrm{~dB}$ for signal to noise ratio, and -49 to $-5 \mathrm{~dB}$ for total noise for 1-260 channels. Here, it is analyzed that recorded values are most suitable for the support of super dense transmitting signals due to suitable power amplification from RAMAN-EDFA-RAMAN hybrid optical amplifier which is the prime concern for super dense communication.

In fact, proposed HOA is the strong tool to maintain the uniform power amplification which uniform power amplification is shown in Fig. 4 for the betterment of transmitting optical signals. Furthermore, eye diagram of the signals are also received after the transmission distance of $65 \mathrm{~km}$ at the receiver section to observe the mitigating impact of crosstalk and fiber nonlinearities in Fig. 5.

\section{Conclusion}

This paper has explored for the first time, best of our knowledge, mitigating effect of crosstalk and fiber nonlinearities for $260 \times 10 \mathrm{Gbps}$ super dense wavelength division multiplexing (SD-WDM) system. RAMAN-EDFA-RAMAN hybrid optical amplifier (HOA) has played the main role to control the dominating effect of optical domain for $65 \mathrm{~km}$ optical communication distance. Recorded outcomes have really supported to the propose system and it has also recommended that this model will be showing massive impact to upgrade the service quality of the network provider for super dense communication. 


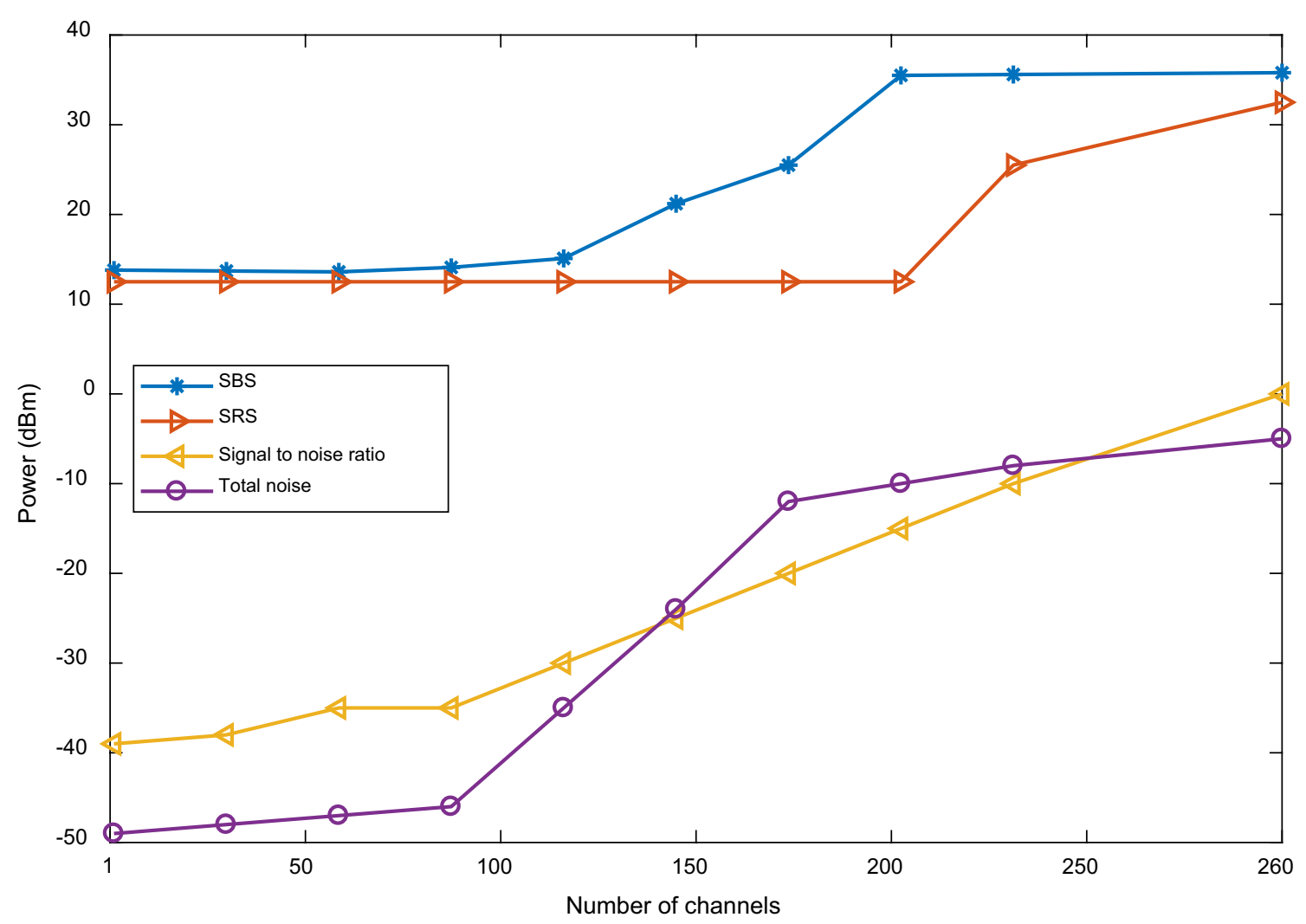

Fig. 3 Power of fiber nonlinearities with respect to number of channels

Fig. 4 Power amplification from RAMAN-EDFA-RAMAN hybrid optical amplifier

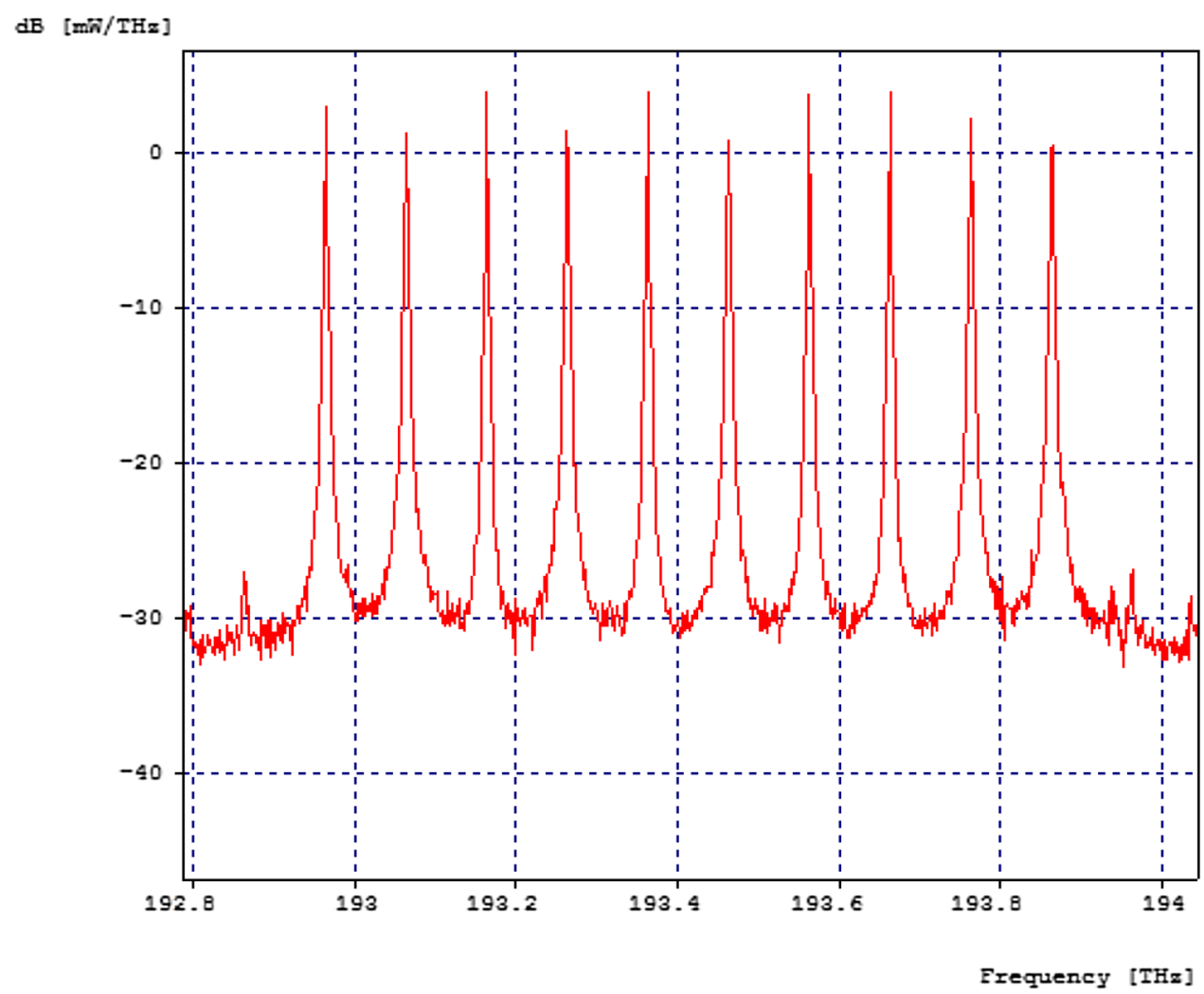


Fig. 5 Eye diagram of received signals at the receiver section after the transmission distance of $65 \mathrm{~km}$

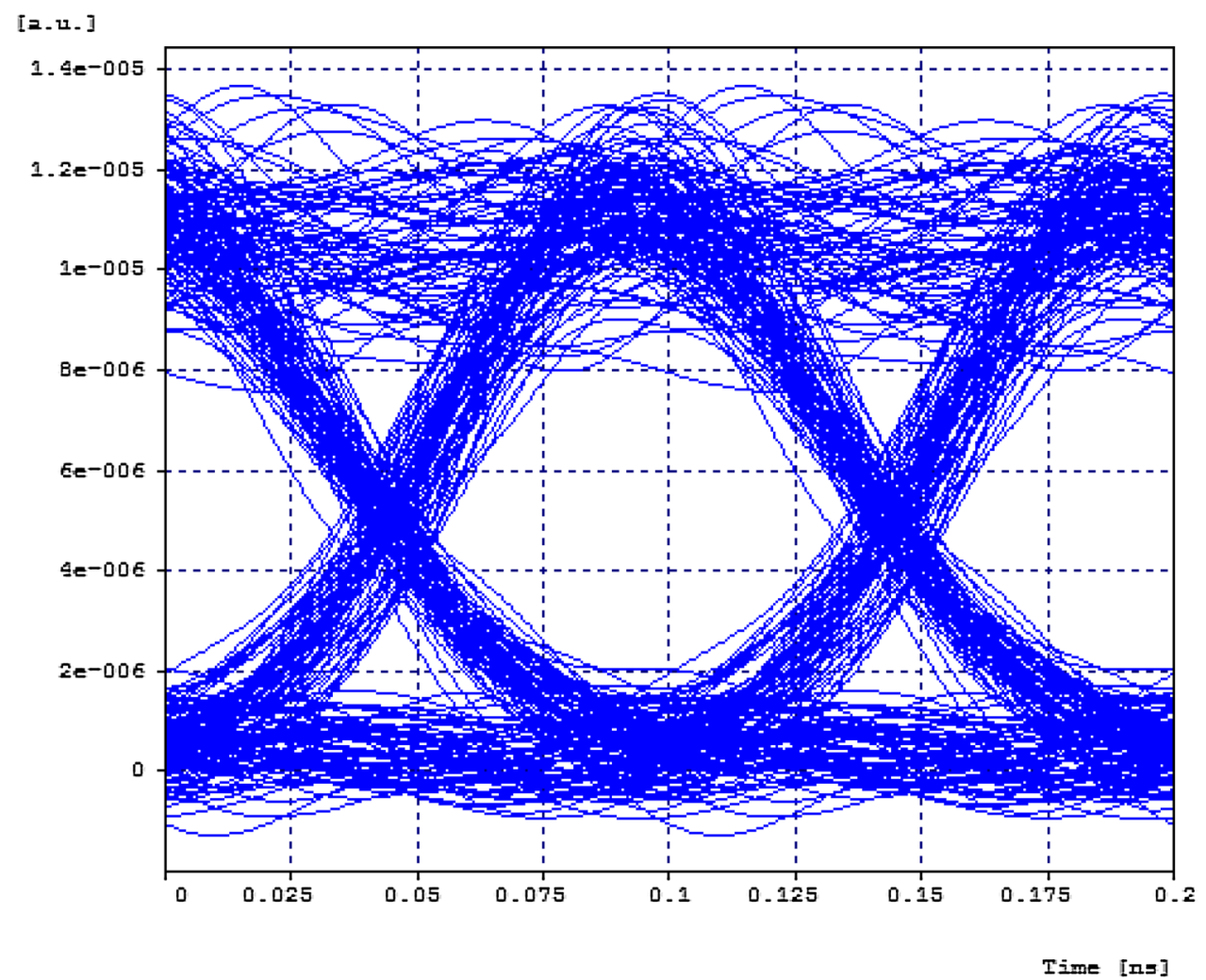

Funding One of the authors (Chakresh kumar) is grateful to Guru Gobind Singh Indraprastha University to provide the fund in terms of FRGS [File No. GGSIPU/DRC/FRGS/2018/70 (1115)] for carried out this research work

\section{Compliance with ethical standards}

Conflict of interest Further, authors are also declared that they have no conflict of interest.

\section{References}

1. Leven A, Kaneda N, Koc U, Chen Y (2007) Frequency estimation in intradyne reception. IEEE Photon Technol Lett 19(6):366-368

2. Zhang F, Wang D, Ding R, Chen Z (2014) Terabit Nyquist PDM32QAM signal transmission with training sequence based time domain channel estimation. Opt Expr 22(19):23415-23426

3. Zhou X (2010) An improved feed-forward carrier recovery algorithm for coherent receiver with M-QAM modulation format. IEEE Photon Technol Lett 22(14):1051-1053

4. Jia Z, Yu J, Chien H, Dong Z, Huo D (2012) Field transmission of $100 \mathrm{G}$ and beyond: multiple baud rates and mixed line rates using Nyquist-WDM technology. J Lightwave Technol 30(24):3793-3804

5. Eliasson H, Johannisson P, Karlsson M, Andrekson P (2015) Mitigation of nonlinearities using conjugate data repetition. J Lightwave Technol 23(3):2392-2402
6. Dar R, Feder M, Mecozzi A, Shtaif M (2013) Properties of nonlinear noise in long, dispersion-uncompensated fiber links. Opt Expr 21(22):25685-25699

7. Secondini M, Forestieri E (2012) Analytical fiber-optic channel model in the presence of cross-phase modulation. IEEE Photon Technol Lett 24(22):2016-2019

8. Secondini M, Forestieri E, Prati G (2013) Achievable information rate in nonlinear WDM fiber-optic systems with arbitrary modulation formats and dispersion maps. J Lightwave Technol 31(23):3839-3852

9. Zheng Z, Ding R, Zhang F, Chen Z (2013) $1.76 \mathrm{~Tb} / \mathrm{s}$ Nyquist PDM 16QAM signal transmission over $714 \mathrm{~km} \mathrm{SSMF}$ with the modified SCFDE technique. Opt Expr 21(15):17505-17511

10. Dar R, Feder M, Mecozzi A, Shtaif M (2015) Inter-channel nonlinear interference noise in WDM systems: modeling and mitigation. J Lightwave Technol 33(5):1044-1053

11. Mecozzi A, Essiambre R (2012) Nonlinear Shannon limit in pseudolinear coherent systems. J Lightwave Technol 30(12):2011-2024

12. Secondini M, Forestieri E (2014) On XPM mitigation in WDM fiber-optic systems. IEEE Photon Technol Lett 26(22):2252-2255

13. Liu X, Chraplyvy A, Winzer P et al (2013) Phase-conjugated twin waves for communication beyond the Kerr nonlinearity limit. Nat Photonics 7:560-568

14. Gao Y, Cartledge J, Karar A et al (2014) Reducing the complexity of perturbation based nonlinearity pre-compensation using symmetric EDC and pulse shaping. Opt Expr 22(2):1209-1219

15. Tao Z, Dou L, Yan W et al (2011) Multiplier-free intrachannel nonlinearity compensating algorithm operating at symbol rate. J Lightwave Technol 29(17):2570-2576

16. Kumar C, Goyal R (2018) Performance analysis of hybrid optical amplifiers for super dense wavelength division multiplexing 
system in the scenario of reduced channel spacing. Mapan 33(2):159-164

17. Kumar C, Goyal R (2018) L-Band flat-gain raman with erbiumdoped fluoride hybrid optical amplifier for superdense wavelength division multiplexing system. J Russ Laser Res 39(3):263-266

18. Kumar C, Goyal R (2018) A novel flattened gain C-band cascaded hybrid optical Raman and thulium-doped fluoride fiber amplifier for super dense wavelength division multiplexing system. Opt Appl 48(2):173-177

Publisher's Note Springer Nature remains neutral with regard to jurisdictional claims in published maps and institutional affiliations. 